\title{
Anti-caries DNA vaccine-induced secretory immunoglobulin A antibodies inhibit formation of Streptococcus mutans biofilms in vitro
}

\author{
Li HUANG, Qing-an XU, Chang LIU, Ming-wen FAN*, Yu-hong LI*
}

Key Laboratory for Oral Biomedical Engineering of Ministry of Education, School \& Hospital of Stomatology, Wuhan University, Wuhan 430079, China

\begin{abstract}
Aim: To investigate the effects of anti-caries DNA vaccine-induced salivary secretory immunoglobulin A (S-IgA) antibodies on Streptococcus mutans (S. mutans) adherence and biofilms formation in vitro.

Methods: Adult female Wistar rats were intranasally immunized with the anti-caries DNA vaccine pGJA-P/VAX. Their saliva samples were collected at different times after the immunization, and S-lgA antibody level in the saliva and its inhibition on S. mutans adherence were examined. The effects of S-IgA in the saliva with the strongest inhibitory effects were examined at 3 different stages, ie acquired pellicles, biofilm formation and production of mature biofilms. The number of viable bacteria and depth of the biofilm at $16 \mathrm{~h}$ in each stage were determined using counting colony forming units and using a confocal laser scanning microscopy (CLSM). The participation of S-IgA in acquired pellicles and its aggregation with S. mutans were also observed under CLSM.

Results: The S-IgA titer in saliva reached its peak and exhibited the strongest inhibition on S. mutans adhesion at 10 weeks after the immunization. The colonies and depth of the biofilm in the saliva-pretreated group were $41.79 \%$ and $41.02 \%$, respectively, less than the control group. The colonies and depth of the biofilm in the co-culture group were $27.4 \%$ and $22.81 \%$ less than the control group. The assembly of S. mutans and S-IgA was observed under CLSM after co-cultivation. In the mature-stage biofilm, no differences were observed between the different groups.

Conclusion: These results demonstrate that the anti-caries DNA vaccine induces the production of specific S-IgA antibodies that may prevent dental caries by inhibiting the initial adherence of S. mutans onto tooth surfaces, thereby reducing the accumulation of S. mutans on the acquired pellicles.
\end{abstract}

Keywords: teeth; dental plaque; dental caries; Streptococcus mutans; biofilm; S-IgA; DNA vaccine

Acta Pharmacologica Sinica (2013) 34: 239-246; doi: 10.1038/aps.2012.145; published online 31 Dec 2012

\section{Introduction}

Streptococcus mutans (S. mutans) is the predominant microorganism in the etiology and pathogenesis of dental caries ${ }^{[1,2]}$. These organisms form dental plaque on the tooth surface, which leads to the development of caries $^{[3]}$. The adherence of $S$. mutans onto the tooth surface is the initial step and a major factor in the formation of dental plaque ${ }^{[4,5]}$. Because S. mutans is the primary bacterial agent of dental caries, the virulent factors by which it adheres to tooth surfaces are important potential targets for anticariogenic intervention.

There are two major virulent factors related to $S$. mutans adherence: the glucosyltransferase (GTF) enzyme and the

\footnotetext{
* To whom correspondence should be addressed.

E-mail liyuhong_an@yahoo.com.cn (Yu-hong LI);

kqyywjtx@public.wh.hb.cn (Ming-wen FAN)

Received 2012-07-02 Accepted 2012-09-24
}

surface protein antigen AgI/II $(\mathrm{PAc})^{[6-9]}$. The GTF enzyme synthesizes glucans from sucrose and mediates the sucrosedependent accumulation of $S$. mutans on the tooth surface ${ }^{[10]}$. One region of the GTF, the glucan-binding domain (GLU) is responsible for the composition of the glucans. Glucans mediate the sucrose-dependent adherence of $S$. mutans and help $S$. mutans tightly adhere to the tooth surface, forming compact dental plaque ${ }^{[11]}$. The PAc protein possesses two important functional and immunogenic regions: an N-terminal region that is alanine-enriched and the middle P-region that is proline-enriched, both of which initialize $S$. mutans adherence to the acquired pellicle on the tooth surface ${ }^{[12]}$. These two virulent factors mediate the adherence of $S$. mutans. To target these factors and fight dental caries, a fusion DNA vaccine called pGJA-P/VAX was constructed using the GLU domain of the GTF enzymes and the A-P fragments of the S. mutans PAC genes. After intranasally immunization of an experi- 
mental animal model, this vaccine elicited a specific S-IgA response in saliva that produced a significant anti-caries effect, as determined by the altered caries scores ${ }^{[1,13,14]}$.

In our previous studies, the targeted fusion DNA vaccine pGJA-P/VAX was administered intranasally in a rat model and demonstrated a significant inverse correlation between the levels of anti-PAc IgA in saliva and the total caries score. In response to $\mathrm{pGJA}-\mathrm{P} / \mathrm{VAX}$ immunization, the secretory IgA inhibits colonization of $S$. mutans, effectively preventing dental caries on rat teeth ${ }^{[15,16]}$. In saliva, S-IgA is the major antibody that acts as the first line of mucosal defense against dental caries by interfering with microbial adherence and bacterial colonization ${ }^{[17]}$. However, there is no direct evidence proving that S-IgA induced by this vaccination prevents S. mutans from adhering to the tooth's surface and forming plaque biofilms, which are the hallmark of the initiation and formation of dental caries. The aim of this study is to investigate the impact of S-IgAs on S. mutans adherence and to identify how S-IgAs are induced by the anti-caries DNA vaccine and how S-IgAs interfere with the pathogenesis of $S$. mutans infections in vitro.

\section{Materials and methods}

\section{Bacteria and growth conditions}

In this study, the following $S$. mutans strains were used: S. mutans MT 8148 and UA140:: $\varphi$ (mutAp-mrfp1) (provided by Kreth, UCLA School of Dentistry); the monomeric red fluorescent protein (mrfp1) exhibits a red fluorescence when analyzed using fluorescence microscopy ${ }^{[18]}$. Both strains of S. mutans were routinely cultured in brain heart infusion (BHI) agar or Modified Scholtens' Broth (MSB) agar at $37^{\circ} \mathrm{C}$ in an atmosphere with $85 \% \mathrm{~N}_{2}, 5 \% \mathrm{H}_{2}$, and $10 \% \mathrm{CO}_{2}$; spectinomycin $(800 \mu \mathrm{g} / \mathrm{mL})$ was added to the medium to select for the transformants carrying the reporter plasmid in the UA140:: $\varphi$ (mutAp-mrfp1) strain. A standard curve of S. mutans concentrations was obtained and used to determine the bacterial concentration levels of the test samples.

\section{Plasmid preparation}

The anti-caries DNA vaccine pGJA-P was constructed as previously described ${ }^{[14]}$. Briefly, pGJA-P encodes the following: the A-P fragment of the pac gene from S. mutans, the signal peptide and extracellular regions of the human CTLA4 gene, the hinge and Fc regions of the human Igr1 gene, and the GLU domain of the $g t f B$ gene from $S$. mutans. The targeted anti-caries DNA plasmid pGJA-P/VAX1 was successfully constructed by ligating pGJA-P into pVAX1 (Invitrogen, Carlsbad, CA, USA). The pVAX1 vector was used as a control. The plasmids were prepared with a commercial EndoFree Plasmid Mega Kit (Qiagen, Hilden, Germany) and stored at $-20^{\circ} \mathrm{C}$. A bupivacaine: DNA complex was prepared by adding bupivacaine hydrochloride to an aqueous DNA solution using a fast-mixing method ${ }^{[19]}$. The final bupivacaine and DNA concentrations were $0.25 \%$ and $1 \mu \mathrm{g} / \mu \mathrm{L}$, respectively.

\section{Rat immunization and sample collection}

Four-to-six-week-old female Wistar rats, eight per group, received intranasal immunizations with either pGJA-P/VAX (Group A) or pVAX1 (Group B) at week 0 and week 2. In each group, $100 \mu \mathrm{g}$ of the bupivacaine:DNA complex was applied to the nostril. To stimulate salivation, the rats received an intraperitoneal injection of $0.2 \%$ pilocarpine (Sigma Chemical Co, St Louis, MO, USA), and their saliva samples were collected at 2, 4, 6, 8, 10, 12, 14, and 16 weeks after their first immunization. Prior to analyzing their antibody activity, the saliva samples were centrifuged and stored at $-70^{\circ} \mathrm{C}$. All of the animal protocols performed in this study were approved by the Hubei Medical Laboratory Animal Center Review Board, Wuhan, China.

\section{Antibody analysis}

An enzyme-linked immunosorbent assay (ELISA) was performed to determine the specific antibody titers against PAc and Glu in the saliva samples, as previously described ${ }^{[16]}$. Briefly, each well of the 96-well microtiter plate (Nunc, Roskilde, Denmark) was coated with recombinant PAc or GLU $(10 \mu \mathrm{g} / \mathrm{mL}$ in carbonate buffer, $\mathrm{pH}$ 9.6) and incubated overnight at $4{ }^{\circ} \mathrm{C}$. Nonspecific binding was blocked by incubating the samples in 3\% bovine serum albumin (BSA) in phosphate buffered saline (PBS) containing $0.05 \%$ Tween 20 (PBST) for $2 \mathrm{~h}$ at $37^{\circ} \mathrm{C}$; then, the plates were washed with PBST at room temperature three times for 5 min each. The saliva was serially diluted (with a starting dilution of 1:2) and added, in duplicate, to individual wells, which were incubated at $37^{\circ} \mathrm{C}$ for $1.5 \mathrm{~h}$. Subsequently, the plates were washed and incubated with a peroxidase-conjugated goat anti-rat IgA (Sigma, St, Louis, MO, USA), at a 1:1000 dilution in blocking buffer, for $2 \mathrm{~h}$ at $37^{\circ} \mathrm{C}$. Then, the o-phenylenediamine substrate was added with $\mathrm{H}_{2} \mathrm{O}_{2}$ and incubated at $37^{\circ} \mathrm{C}$ for 30 min; the reaction was stopped using $2 \mathrm{~mol} / \mathrm{L} \mathrm{H}_{2} \mathrm{SO}_{4}$, and the optical density at $490 \mathrm{~nm}\left(O D_{490}\right)$ was recorded. The antibody titer was defined as the reciprocal of the highest dilution with an $O D_{490}$ of 0.1 above the blank control. The saliva with the highest antibody concentration level was chosen for the next experiments.

\section{The adherence inhibition assay}

The effect of S-IgA antibodies on the adhesion of S. mutans MT8148 to S-HA beads was examined as previously described ${ }^{[20]}$. Briefly, $5 \mathrm{mg}$ of HA beads were equilibrated in buffered $\mathrm{KCl}$ at room temperature overnight. The experimental pellicles were prepared by treating the beads with $125 \mu \mathrm{L}$ of normal rat saliva at room temperature for $1 \mathrm{~h}$ while continuously inverting the mixture at $6 \mathrm{r} / \mathrm{min}$. After washing twice with buffered $\mathrm{KCl}$, the $\mathrm{HA}$ beads were treated for $30 \mathrm{~min}$ with buffered $\mathrm{KCl}$, which contained BSA $(5 \mathrm{mg} / \mathrm{mL})$ to block any uncoated regions of the HA. After two washes with buffered $\mathrm{KCl}$, the treated beads were incubated for $1 \mathrm{~h}$ with $\left[{ }^{3} \mathrm{H}\right]$ thymidine-labeled S. mutans cells $\left(2.0 \times 10^{9}\right.$ cells $)$ suspended in 100 $\mu \mathrm{L}$ of buffered $\mathrm{KCl}$ containing BSA $(5 \mathrm{mg} / \mathrm{mL})$ and $100 \mu \mathrm{L}$ of 4-week, 10-week, and 16-week saliva, and $100 \mu \mathrm{L}$ of the serial diluted saliva, for the 10-week immunized mice $(1: 2 ; 1: 4 ; 1: 8$; $1: 16 ; 1: 32)$. The saliva from the unimmunized mice and PBS 
was used as the control. The beads were washed three times with buffered $\mathrm{KCl}$, and the bound radioactivity was measured using a liquid scintillation spectrometer (TRICARB-2000A; Packard Instruments, Downers Grove, IL, USA). The number of streptococcal cells attached to the beads was determined from the calculated specific radioactivity of the bacteria.

\section{The preparation of $S$. mutans biofilms}

S. mutans biofilms [UA140:: $\varphi$ (mutAp-mrfp1)] were formed on saliva-coated hydroxyapatite discs; to grow the $S$. mutans biofilms, a technique described by Fraud et al was modified ${ }^{[21]}$ $S$. mutans was cultured on sintered circular ceramic HA disks (9.8 $\mathrm{mm}$ in diameter $\times 1.5 \mathrm{~mm}$ in height; Clarkson Chromatography Products, South Williamsport, PA, USA) that had been incubated with saliva from rats that had not been immunized. The saliva to cultivate the biofilms was pre-filtered through a $5-\mu \mathrm{m}$ and then a $0.45-\mu \mathrm{m}$ filter (Fisher Scientific, Loughborough, UK). The sterilized HA disks were placed on cell culture inserts in Costar 24-well plates and incubated with $0.5 \mathrm{~mL}$ of sterile saliva under agitation $(120 \mathrm{r} / \mathrm{min})$ for $4 \mathrm{~h}$ at $37^{\circ} \mathrm{C}$. After the formation of a saliva pellicle, the saliva was removed by aspiration and replaced with $0.8 \mathrm{~mL}$ of $\mathrm{BHI}$, $0.15 \%(w / v)$ sucrose, and $0.8 \mathrm{~mL}$ sterile saliva. Each well was inoculated with a suspension of $S$. mutans $(0.2 \mathrm{~mL}$, containing $10^{8} \mathrm{CFU} / \mathrm{mL}$ ), and the culture plates were incubated anaerobically at $37^{\circ} \mathrm{C}$ for $16 \mathrm{~h}$. At the end of the incubation period, the HA disks were dip-washed twice in PBS and gently swirled to remove any loosely adherent bacteria. To harvest the adherent cells, each disk was transferred to a sterile $50 \mathrm{~mL}$ polypropylene tube containing $1 \mathrm{~mL}$ of diluent and was vigorously vortexed for $2 \mathrm{~min}$. Then, the fluid containing the detached cells was vortexed for $1 \mathrm{~min}$ with glass beads to disaggregate any clumped cells. After anaerobic inoculation for $48 \mathrm{~h}$, the total numbers of $S$. mutans colonies were counted on the MSB plates. S. mutans was identified by its characteristic appearance.

Scanning electron microscopy (SEM) investigation of the biofilms SEM determined the topography of the HA disk surface, the formation of bacterial biofilms on HA disks, and the efficiency of the resuspension methods. The HA disks were fixed using $2.5 \%(v / v)$ glutaraldehyde for $2 \mathrm{~h}$ at $4{ }^{\circ} \mathrm{C}$ after cultivation. After two washes in deionized water, the disks were dehydrated by incubation in 25\% ethanol for $15 \mathrm{~min}, 50 \%$ ethanol for $15 \mathrm{~min}$, $75 \%$ ethanol for $15 \mathrm{~min}$, and $100 \%$ ethanol for $15 \mathrm{~min}$. The last step was performed twice. The disks were air-dried overnight, coated with gold and observed under a SEM (Quanta 600, FEI company, Holland).

\section{Confocal laser scanning microscopy (CLSM) investigation}

Confocal laser scanning microscopy (CLSM) was used to determine the depth of the biofilms and the interactions between the S-IgA and S. mutans. The S. mutans biofilms were grown for $16 \mathrm{~h}$. At the end of the experimental period, the biofilms were removed and subjected to washing and swirling to remove the loosely adherent bacteria. For the biofilm observations, a CLSM fitted with a water-immersion dipping lens $(10 \times)$ was used, and the biofilm structure was analyzed as a series of horizontal opto-digital sections, each $1.5 \mu \mathrm{m}$ thick.

\section{The effect of S-IgA pretreated HA disks on biofilm formation} The HA disks were incubated at $37^{\circ} \mathrm{C}$ for $4 \mathrm{~h}$ with $500 \mu \mathrm{L}$ of saliva from groups A and B under agitation (120 r/min). After an acquired pellicle formed, the saliva was removed by aspiration and replaced with $0.8 \mathrm{~mL} \mathrm{BHI}, 0.15 \%(w / v)$ sucrose, S. mutans $\left(0.2 \mathrm{~mL}\right.$, containing $\left.10^{8} \mathrm{CFU} / \mathrm{mL}\right)$, and $0.8 \mathrm{~mL}$ sterile blank saliva. The biofilms were grown for $16 \mathrm{~h}$ before the cells were harvested and enumerated, and the depths of the biofilms were measured, as described above. The acquired pellicles formed on the HA disks were incubated with a speciesmatched goat-anti-rat IgA:FITC antibody (AbD, MorphoSys UK Ltd, Oxford, UK) in PBS containing 1\% bovine serum albumin (BSA). After $1 \mathrm{~h}$ of incubation at room temperature, the HA disks were washed three times with PBS for 5 min and then observed using the CLSM to investigate the distribution of S-IgA and bacteria on the HA disks.

\section{The effect of S-IgA on S. mutans adherence to the acquired pellicles}

The acquired pellicles were formed on the HA disks with 500 $\mu \mathrm{L}$ of blank saliva for $4 \mathrm{~h}$ under agitation. Then, the saliva was replaced with $0.8 \mathrm{~mL}$ BHI, $0.15 \%(w / v)$ sucrose, $S$. mutans $\left(0.2 \mathrm{~mL}\right.$, containing $\left.10^{8} \mathrm{CFU} / \mathrm{mL}\right)$, and $0.8 \mathrm{~mL}$ sterile saliva from either the experimental or control groups. The $S$. mutans biofilms were cultivated for $16 \mathrm{~h}$; the cells were recovered and enumerated, and the depth was measured as described above. After cultivation, the planktonic cells were collected from the 24-well polystyrene culture plates, transferred into 5-mL polypropylene tubes, and centrifuged for $5 \mathrm{~min}(451 \times g)$. The deposition was washed three times with PBS and incubated with species-matched anti-IgA antibody in PBS containing 1\% BSA. After incubation for $1 \mathrm{~h}$ at $37^{\circ} \mathrm{C}$, the deposition was washed with PBS. The confocal microscope was equipped with detectors and filter sets to monitor FITC (green) and mRfp (red) fluorescence; the merged images depict the binding of $S$. mutans and S-IgA in the deposition.

\section{The activity of the S-IgA in mature biofilms}

The $S$. mutans biofilms were cultivated with blank saliva for $16 \mathrm{~h}$, as described above. The HA disks supporting the biofilms were washed and transferred to new wells containing $1.0 \mathrm{~mL}$ of sterile saliva from either the experimental or control groups. After $1 \mathrm{~h}$ of contact at room temperature, the disks were washed. The surviving bacteria were resuspended as described above, the viable bacteria were counted and the biofilm was measured, as described.

\section{Statistical analysis}

SPSS 12.0 software (SPSS, Inc, Chicago, IL, USA) was used for the statistical analyses. The differences in the antibody titers among the groups, the depth of the biofilms, and the viable bacteria on the biofilms were determined using a one-way 
analysis of variance (ANOVA). A value of $P<0.05$ was considered significant.

\section{Results}

\section{Antibody analysis}

The kinetics of the salivary IgA antibody were investigated, as shown in Figure 1. The salivary anti-PAc and anti-Glu IgA responses in rats treated with the DNA vaccination were all detected at 4 weeks after the second immunizations and persisted until the end of the experiment (16 weeks); these responses were significantly higher $(P<0.01, P<0.05))$ than those in the vector-immunized rats. The IgA anti-PAc and anti-Glu responses in rats peaked at 10 weeks, and these samples were chosen for the following experiments. In the control group, no effective salivary IgA responses were observed.

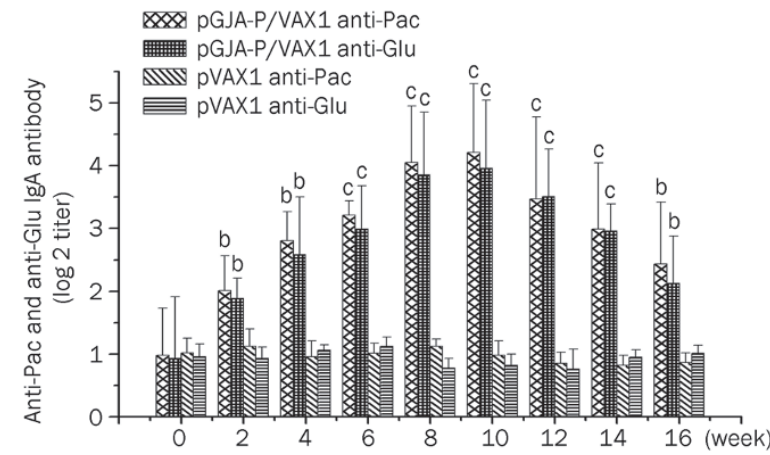

Figure 1. The specific secretory anti-Pac and anti-Glu S-IgA antibody levels detected in individual rats after immunization with pGJA-P/VAX or pVAX1. Mean \pm SD. $n=3 .{ }^{b} P<0.05,{ }^{c} P<0.01$ vs 0 week.

\section{Inhibition of S. mutans adherence}

The adherence inhibition analyses of the salivary samples from the immunized rats at different time points and at the serial dilutions are depicted in Figure 2. The adherence of $S$. mutans in the saliva of the immunized rats at each time point was significantly lower than the control group $(P<0.001)$. The S-IgA antibodies in the 10th week showed the strongest inhibition of $S$. mutans cell adhesion, but there were no significant differences in this parameter between the $4 \mathrm{th}$, 10th, and 16th weeks (Figure 2A). The salivary samples containing specific S-IgA also inhibited $S$. mutans binding in a dose-dependent manner; the lowest saliva dilution with an effect was 1:16 and this concentration reduced the adherence activity of $S$. mutans to approximately $8.7 \%$ (Figure 2B).

\section{An SEM investigation of S. mutans biofilms on HA disks}

The protocol used for growing $S$. mutans biofilms on salivacoated HA disks was highly reproducible and with a low variability between the separate experiments. After incubation, the $S$. mutans biofilm uniformly covered the surface of the HA disks (observed under SEM). The protocol to remove the adherent bacteria for enumeration was highly efficient because
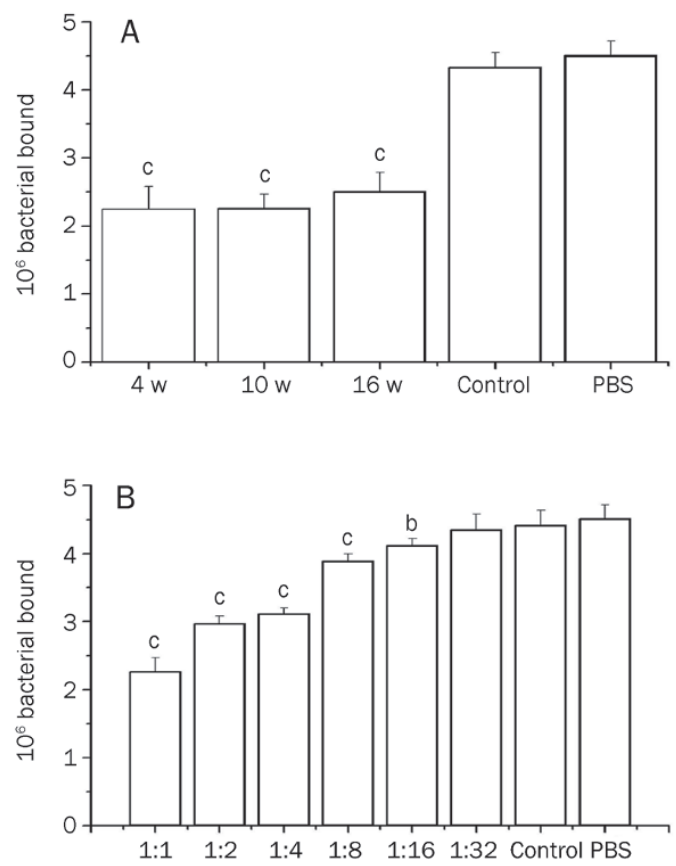

Figure 2. Adherence inhibition analysis of salivary sample. (A) The adherence of $\mathrm{S}$. mutans at different time points was significantly lower in saliva of immunized rats than that in the control group, but there were no significant differences between the 4th, 10th, and 16th week. (B) Different dilutions of saliva could inhibit the adherence of $S$. mutans, and the lowest dilution with effect was $1: 16$. Mean \pm SD. $n=3$. ${ }^{\mathrm{b}} P<0.05$, ${ }^{c} P<0.01$ vs control.

very few cells remained attached to the surface of the HA disks when observed under SEM (Figure 3).

The effect of the S-IgA pretreated HA disks on biofilm formation When the HA disks were pretreated with S-IgA saliva before pellicle formation, the formation of $S$. mutans biofilms was prevented; more S-IgAs (Figure 4, green) formed on the pellicle using the saliva from group A, compared with the pellicle formed by group B. There were significant differences in the number of cells recovered from the biofilm and the depth of the biofilm compared to the control group $(P<0.01$; Table 1$)$. There were $41.79 \%$ fewer cells in the pellicle formed by group A, and the biofilm was $41.02 \%$ less deep than that formed after saliva pretreatment with group $B$.

Table 1. Effect of S-IgA pre-treatment of HA disks on biofilm formation. Analysis of depth of biofilm and $\log 10 \mathrm{CFU} / \mathrm{mL}$ was carried out to compare the differences between the two groups after pre-treating by saliva of experimental (A) or control group (B). Mean \pm SD. $n=8 .{ }^{c} P<0.01$ vs control.

\begin{tabular}{ccc}
\hline & Depth of biofilm $(\mu \mathrm{m})$ & Log10 CFU $/ \mathrm{mL}$ \\
\hline A & $17.66 \pm 3.23^{\mathrm{c}}$ & $4.30 \pm 0.21^{\mathrm{C}}$ \\
B & $30.34 \pm 2.46$ & $7.24 \pm 0.16$ \\
\hline
\end{tabular}

CFU, colony-forming units. 

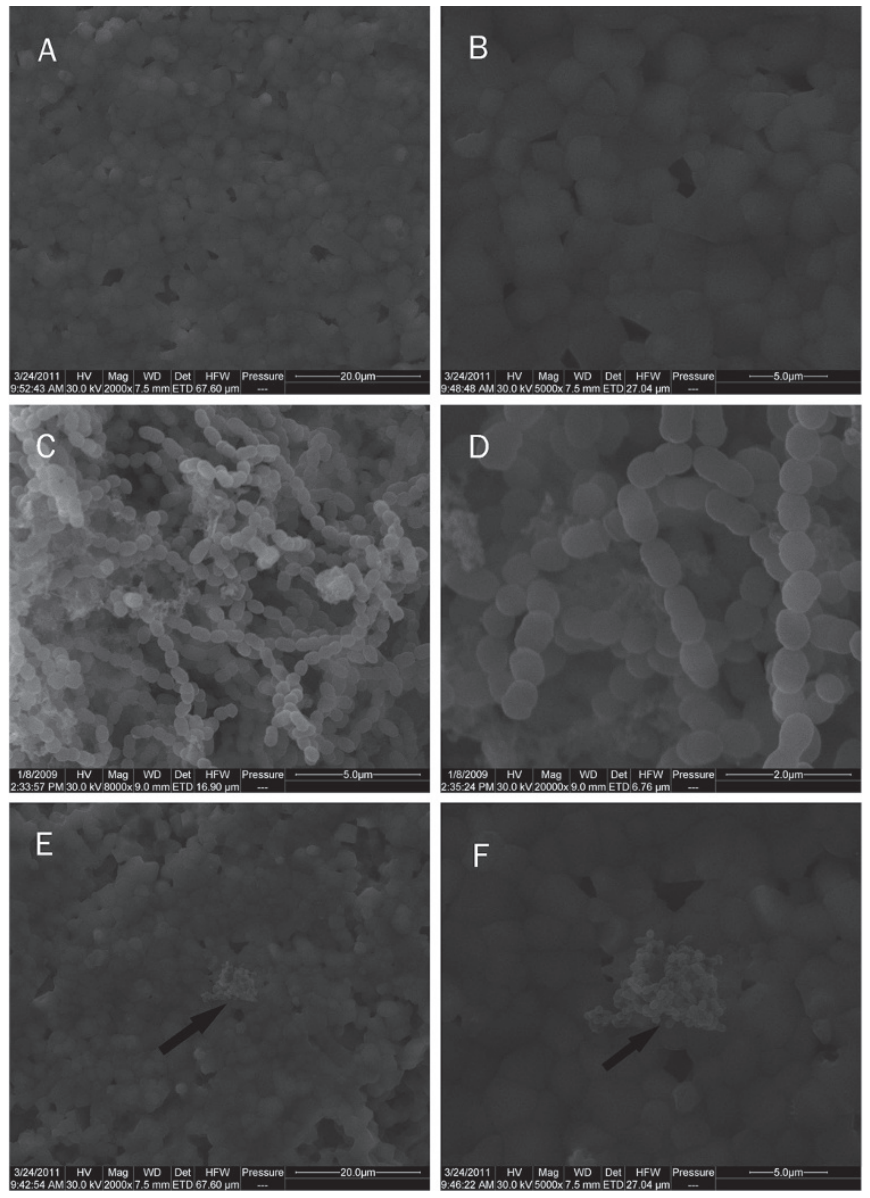

Figure 3. Scanning electron microscopy (SEM) investigation. Surface of hydroxyapatite disks at $2000 \times$ (A) and $5000 \times$ (B) magnification (HA disk was a standard hexagonal structure). S. mutans $16 \mathrm{~h}$ biofilm at $8000 \times$ (C) and $20000 \times$ (D) magnification (S. mutans biofilm uniformly covered the surface of the HA disks). The surface of the hydroxyapatite disks at $2000 \times(E)$ and $5000 \times(F)$ magnification after biofilm resuspension (few remaining bacteria were observed: arrows).
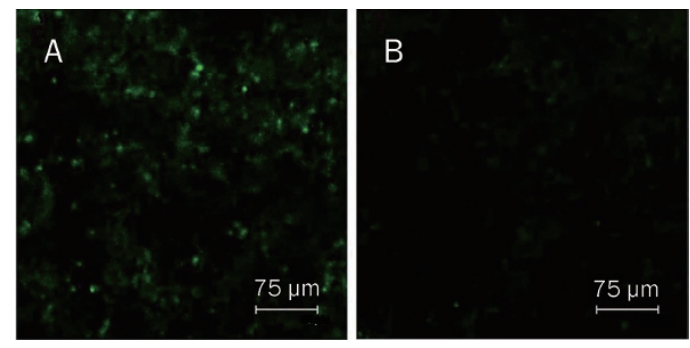

Figure 4. Effect of S-IgA pretreated HA disks on biofilm formation. Acquired pellicle formed by saliva from groups A and B. The pellicle formed by saliva from group A could be observed to have more S-lgA (green) on the HA disks than the pellicle formed by saliva from group B.

The effect of the S-IgA on the S. mutans biofilm formations on the HA disks

The cocultivation of the saliva from the experimental group with the $S$. mutans reduced the bacterial adherence to the
HA disk pellicle (Table 2). The bacteria count from the HA disks $(27.4 \%$ less $)$ and the experimental group (22.81\% less) were less than those in the biofilms obtained from the control group $(P<0.01)$. Two fluorescence images $(\mathrm{S}-\mathrm{IgA}$ : green and S. mutans: red) were taken from the same microscopic field, and then the images were merged. S-IgA and S. mutans binding was observed with the confocal microscope: the green fluorescence was around or superposed with the red fluorescence, suggesting that the S-IgA can assemble the S. mutans or cover the surface of the S. mutans (Figure 5). Meanwhile, the biofilm from group B had no such appearance. Fewer S. mutans and a thinner biofilm in the experimental group demonstrated that the S-IgA prevented the adherence of the S. mutans to the HA disks.

Table 2. Effect of S-IgA on the adherence of S. mutans onto the acquired pellicles. Analysis of depth of biofilm and Log $10 \mathrm{CFU} / \mathrm{mL}$ was carried out to compare the differences between the two groups after co-cultivated with saliva of experimental (A) or control group (B). Mean \pm SD. $n=8$. ${ }^{c} P<0.01$ vs control.

\begin{tabular}{ccc}
\hline & Depth of biofilm $(\mu \mathrm{m})$ & Log10 CFU $/ \mathrm{mL}$ \\
\hline A & $24.36 \pm 1.49^{\mathrm{c}}$ & $6.09 \pm 0.19^{\mathrm{c}}$ \\
B & $33.56 \pm 1.98$ & $7.89 \pm 0.25$ \\
\hline
\end{tabular}

CFU, colony-forming units.

The activity of the S-IgA against mature biofilms

The S-IgA did not have an effect on the $16 \mathrm{~h}$ mature S. mutans biofilms (Table 3), and there were no significant differences in the viability between the bacteria from the biofilms and the depth of the biofilms treated with either group A saliva, group B saliva, or PBS.

Table 3. Activity of S-lgA against mature biofilms. Analysis of depth of biofilm and Log10 $\mathrm{CFU} / \mathrm{mL}$ was carried out to compare the differences between the three groups after mature biofilms treatments by saliva of experimental (A), control groups (B), or PBS. Mean \pm SD. $n=8$. ${ }^{a} P>0.05$ vs control.

\begin{tabular}{lcl}
\hline & Depth of biofilm $(\mu \mathrm{m})$ & Log10 CFU $/ \mathrm{mL}$ \\
\hline A & $31.52 \pm 3.21^{\mathrm{a}}$ & $7.31 \pm 0.09^{\mathrm{a}}$ \\
B & $32.31 \pm 2.16$ & $7.52 \pm 0.12$ \\
PBS & $32.36 \pm 1.10$ & $7.48 \pm 0.23$ \\
\hline
\end{tabular}

CFU, colony-forming units.

\section{Discussion}

S. mutans has been implicated as one of the principal etiological agents in the pathogenesis of dental caries ${ }^{[22,23]}$. A targeted fusion DNA vaccine encoding the pac and glu genes of $S$. mutans was constructed and demonstrated an anti-caries effect. In the current study, rats were immunized with a 


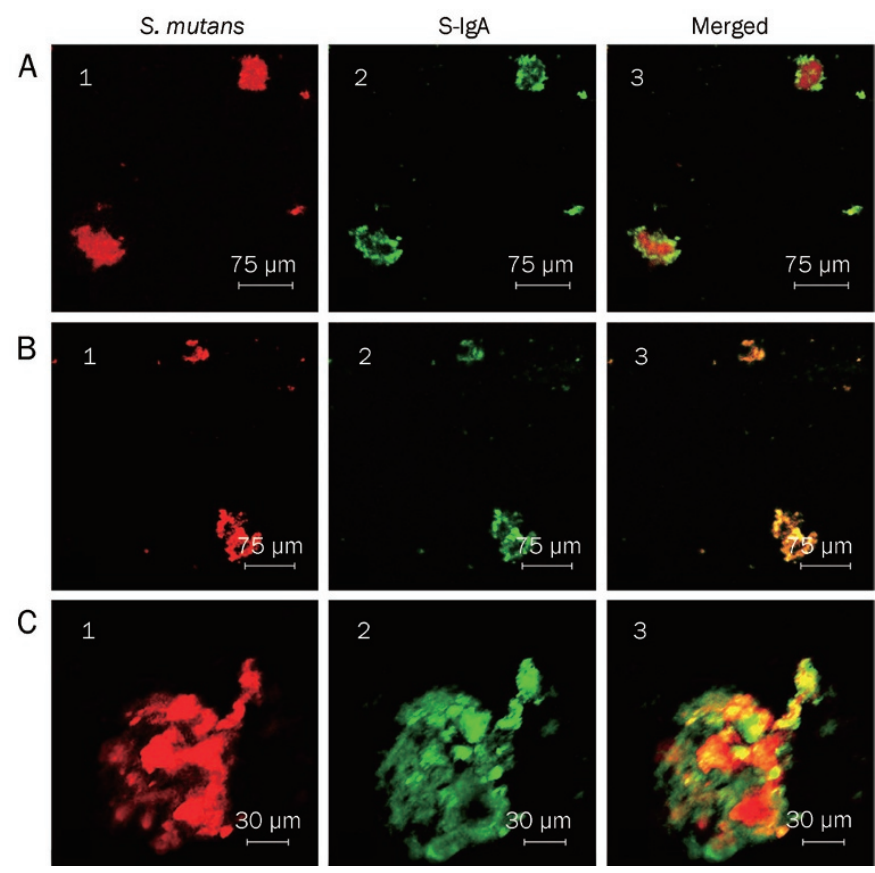

Figure 5. Confocal microscopic image showing distribution of S-IgA and S. mutans in depositon after co-cultivating saliva containing S-IgA and S. mutans onto acquied pellicles. Two fluorescence images were taken from the same microscopic field. 1: Fluorescence imaging of S. mutans (red); 2: Fluorescence imaging of S-IgA (green); 3: Merged fluorescence imaging of 1 and 2. (A) The green fluorescence generally presents around the red fluorescence, indicating S-IgA could assemble S. mutans together. (B) The green fluorescence could be seen superposed with the red fluorescence, indicating S-IgA could cover the surface of S. mutans. (C) The green fluorescence is around or superposed with the red fluorescence, demonstrating S-lgA distributes around and among S. mutans, indicating S-IgA may have the effect by both covering and assembling the $S$. mutans.

targeted anti-caries DNA plasmid and the kinetics of the salivary IgA antibody response was evaluated by ELISA. Then, the salivary samples containing the highest levels of the IgA antibody were collected. The effects of the S-IgA on the early and late $S$. mutans HA disks adherence were tested and the S-IgA's effect on mature, cultivated S. mutans biofilms was tested using a biofilm model. Our results demonstrate that the targeted fusion anti-caries DNA vaccine induced S-IgA production, which reduced the formation of S. mutans' biofilms. S-IgAs prevented S. mutans pellicle adherence by assembling the $S$. mutans together or by covering the S. mutans surface, which inhibited S. mutans-clustering and the formation of biofilms. However, the S-IgA had no positive effect on mature biofilms.

To identify the immune component of S-IgA in saliva that is responsible for S. mutans adhesion inhibition, the conventional DNA vaccine immunization strategy was used to obtain saliva which contained the targeted immune component. We observed the kinetics of the antibody responses generated following intranasal delivery of pGJA-P/VAX in rats. Here, the kinetics of the antibody responses were consistent with those observed in a previous study from our group ${ }^{[16]}$. The specific S-IgA in the saliva of pGJA-P/VAX-immunized rats increased after 4 weeks and achieved a peak around week 10 . Then, the levels of S-IgA remained high until week 16. To determine the S-IgA antibody levels at each time point and the specific IgA titer that inhibits S. mutans-HA binding, we investigated the effects of $S$. mutans adherence in salivary samples at 4,6 , and 10 weeks and in serial dilutions from 1:1 to 1:32 after 10 weeks. The salivary samples exhibited more adherence inhibition during the 10th week compared with any other time point. There was a significant reduction in the adherence when the 10-week saliva was diluted 1:16. Thus, only the 10 week saliva with the highest concentration of anti-PAc IgA and anti-Glu IgA was chosen to investigate the inhibitory effects of S-IgA on S. mutans biofilm formation. Nevertheless, additional research is necessary to determine the specific IgA titer that will inhibit S. mutans biofilm formation.

To evaluate the efficacy of S-IgA on the formation of biofilms, we used a more reproducible monoculture system to obtain S. mutans biofilms on HA disks. We simulated the tooth surface, which under SEM appears as a standard hexagonal hydroxyapatite (the main component of tooth enamel). The protocol to grow $16 \mathrm{~h} \mathrm{~S}$. mutans biofilms on HA disks is highly reproducible and the $16 \mathrm{~h}$ biofilm was used because normally oral biofilms do not develop beyond this time. This $16 \mathrm{~h}$ biofilm model produces biofilms with reproducible characteristics because this time frame represents the typical interval between tooth brushing.

S. mutans biofilm formation on saliva-coated surfaces is a multistep process ${ }^{[24]}$. The first step is the formation of the acquired pellicle, which is composed of mucin, proteins, IgA, and glycoproteins from the host's saliva. This film immediately forms after cleaning and directly influences the pattern of the initial microbial colonization and the formation of the saliva pellicle. The saliva pellicle affects bacterial adherence and plaque formation ${ }^{[25,26]}$. The second step is the assembly of bacteria on the pellicle. This is mediated by a variety of host receptors, partner bacteria, and specific molecules on the bacterial surface (adhesins). Some of these surface bacteria inhibit planktonic bacteria binding. Meanwhile, other surface bacteria, if attached to the teeth or to other cells, boost bacterial binding. For instance, the GTF enzyme and the surface protein antigen AgI/II (PAc) are some of the major virulent factors for S. mutans ${ }^{[27-29]}$. The last step of the biofilm formation process is the maturation of the biofilm. Here, we explored the mechanisms and the effect of S-IgA on S. mutans biofilms by studying the effects of S-IgA: during pellicle formation; during the initial S. mutans adherence; during the adherence of $S$. mutans onto the formed pellicle; during already mature biofilms. The preventative effects of S-IgA on S. mutans adherence are evident from the changes in CFU and biofilm thickness after cultivation. The results from the three different stages indicate that the initial stage had the smallest biofilm (Table 1), while the maximum biofilm was found in the maturation stage (Table 3). It is interesting to note that the S-IgA for the anti-PAc and anti-Glu antibodies induced by the DNA vaccine 
formed the pellicle, as evidenced by the stronger fluorescence of anti-IgA in the CLSM (Figure 4). It has been speculated that surface proteins of $S$. mutans participate in bacterial adherence to teeth via interactions with the salivary pellicle ${ }^{[8]}$. These antibodies in the obtained pellicles could occupy the site of the salivary pellicle and inhibit the initial adherence of $S$. mutans. This investigation also demonstrated that S-IgA assembles S. mutans together or covers the S. mutans surface (Figure 5). The specific binding of the anti-PAc antibody and PAc on the surface of $S$. mutans contributed to cell aggregation and prevented S. mutans from adhering to formed pellicles. As for the effect of IgA on the mature biofilm, the salivary samples were added after pellicle formation. During this phase, the anti-PAc and anti-Glu antibodies could not kill or inhibit the growth of the S. mutans that had already adhered to the HA surface; therefore, under these conditions there were no significant differences between the saliva with or without the specific IgA antibody.

These results are in line with previous studies that report the effects of S-IgA on S. mutans adherence ${ }^{[30-32]}$. Other reports also demonstrate that purified S-IgA in human milk can inhibit the adhesion of streptococci mutans to saliva-coated HA disks ${ }^{[3,34]}$. Although several papers have reported that S-IgA may contribute to the formation of biofilms and boost the adherence of bacteria ${ }^{[35]}$, it is important to note that all of the S-IgA used in these previous studies focused on the original S-IgA in the host's saliva or serum, while the S-IgA investigated in our study was induced by the targeted anticaries DNA plasmid pGJA-P/VAX1. This is the first study of specific S-IgA-mediated biofilm formation. It provides direct evidence (CLSM images) that anti-PAc and anti-Glu S-IgA, induced by an anti-caries DNA vaccine, affect the formation of S. mutans biofilms. Currently, many researchers focus on the binding of S. mutans and S-IgA. For example, Smith et al reported that the saliva of caries-free subjects includes a significant amount of IgA antibody against $S$. mutans antigen $\mathrm{I} / \mathrm{II}^{[36]}$. Meanwhile, Jespersgaard et al demonstrated that there is a high-molecular weight glycoprotein-amylase complex in saliva that is capable of inhibiting GTF, namely, the GLU domain, and may contribute to the control of S. mutans colonizing the oral cavity ${ }^{[37]}$. We assume S-IgA uses the HA disk surface as an inhibitory mechanism that occupies the binding site of $S$. mutans, which prevents the adherence of $S$. mutans to the HA disks. In the later adherent stage, the S-IgA of antiPAc can block the S. mutans surface protein PAc, which is the main receptor that mediates the mutual assembly of $S$. mutans, and hence prevents the adherence of $S$. mutans onto HA disks or acquired pellicles. The S-IgA of anti-Glu can bind the GLU domain of S. mutans, which is responsible for the synthesis of glucans, the main factor mediating the sucrose-dependent adherence of $S$. mutans, and thus inhibits the formation of biofilms.

In conclusion, the specific anti-PAc and anti-Glu S-IgA induced by the targeted anti-caries DNA vaccine pGJAPVAX1 prevented both early and late $S$. mutans adherence by forming the saliva pellicle or by assembling $S$. mutans together in vitro. Here, only the macroscopic effect of S-IgA was studied, but the detailed effects and mechanisms are unclear. The biofilm model is a very limited simulation of the mouth's environment. This model did not take into account saliva flushing, the mechanical brushing of foods, tooth brushing, the cooperation of S. mutans with other bacteria that cause caries, and the effects of swallowing. Further research is required to build a better fluid model that more accurately simulates the environment of the mouth; there is also a need for further studies to provide direct evidence of an in vivo effect of the specific S-IgA.

\section{Acknowledgements}

This work was financially supported by grants from the National Natural Science Foundation of China (№ 30973312 and 81070822).

\section{Author contribution}

Ming-wen FAN designed the research and revised the paper; Li HUANG performed the experiment; Qing-an XU contributed new reagents or analyzed tools; Chang LIU analyzed the data; Li HUANG and Yu-hong LI wrote the paper.

\section{References}

1 Guo JH, Jia R, Fan MW, Bian Z, Chen Z, Peng B. Construction and immunogenic characterization of a fusion anti-caries DNA vaccine against PAc and glucosyltransferase I of Streptococcus mutans. J Dent Res 2004; 83: 266-70.

2 Hamada S, Slade HD. Biology, immunology, and cariogenicity of Streptococcus mutans. Microbiol Rev 1980; 44: 331-84.

3 Kolenbrander PE. Oral microbial communities: biofilms, interactions, and genetic systems. Annu Rev Microbiol 2000; 54: 413-37.

4 Shimotoyodome A, Kobayashi H, Tokimitsu I, Hase T, Inoue T, Matsukubo T, et al. Saliva-promoted adhesion of Streptococcus mutans MT8148 associates with dental plaque and caries experience. Caries Res 2007; 41: 212-8.

5 Thomas JG, Nakaishi LA. Managing the complexity of a dynamic biofilm. J Am Dent Assoc 2006; 137 Suppl: 10S-15S.

6 Bowen WH, Schilling K, Giertsen E, Pearson S, Lee SF, Bleiweis A, et al. Role of a cell surface-associated protein in adherence and dental caries. Infect Immun 1991; 59: 4606-9.

7 Koga T, Asakawa H, Okahashi N, Hamada S. Sucrose-dependent cell adherence and cariogenicity of serotype c Streptococcus mutans. J Gen Microbiol 1986; 132: 2873-83.

8 Koga T, Okahashi N, Takahashi I, Kanamoto T, Asakawa H, Iwaki M. Surface hydrophobicity, adherence, and aggregation of cell surface protein antigen mutants of Streptococcus mutans serotype c. Infect Immun 1990; 58: 289-96.

9 Yamashita Y, Bowen WH, Burne RA, Kuramitsu HK. Role of the Streptococcus mutans gtf genes in caries induction in the specificpathogen-free rat model. Infect Immun 1993; 61: 3811-7.

10 Kuramitsu HK. Virulence factors of mutans streptococci: role of molecular genetics. Crit Rev Oral Biol Med 1993; 4: 159-76.

11 Monchois V, Willemot RM, Monsan P. Glucansucrases: mechanism of action and structure-function relationships. FEMS Microbiol Rev 1999; 23: 131-51.

12 Russell MW, Mansson-Rahemtulla B. Interaction between surface protein antigens of Streptococcus mutans and human salivary components. Oral Microbiol Immunol 1989; 4: 106-11. 
13 Fan MW, Bian Z, Peng ZX, Zhong Y, Chen Z, Peng B, et al. A DNA vaccine encoding a cell-surface protein antigen of Streptococcus mutans protects gnotobiotic rats from caries. J Dent Res 2002; 81: 784-7.

14 Jia R, Guo JH, Fan MW, Bian Z, Chen Z, Fan B, et al. Immunogenicity of CTLA4 fusion anti-caries DNA vaccine in rabbits and monkeys. Vaccine 2006; 24: 5192-200.

15 Li YH, Huang S, Du M, Bian Z, Chen Z, Fan MW. Immunogenic characterization and protection against Streptococcus mutans infection induced by intranasal DNA prime-protein boost immunization. Vaccine 2010; 28: 5370-6.

16 Xu QA, Yu F, Fan MW, Bian Z, Chen Z, Peng B, et al. Protective efficacy of a targeted anti-caries DNA plasmid against cariogenic bacteria infections. Vaccine 2007; 25: 1191-5.

17 Kilian M, Mestecky J, Russell MW. Defense mechanisms involving Fc-dependent functions of immunoglobulin A and their subversion by bacterial immunoglobulin A proteases. Microbiol Rev 1988; 52 : 296-303.

18 Kreth J, Merritt J, Bordador C, Shi W, Qi F. Transcriptional analysis of mutacin I (mutA) gene expression in planktonic and biofilm cells of Streptococcus mutans using fluorescent protein and glucuronidase reporters. Oral Microbiol Immunol 2004; 19: 252-6.

19 Pachuk CJ, Ciccarelli RB, Samuel M, Bayer ME, Troutman RD, Zurawski DV, et al. Characterization of a new class of DNA delivery complexes formed by the local anesthetic bupivacaine. Biochim Biophys Acta 2000; 1468: 20-30.

$20 \mathrm{Li} \mathrm{YH}$, Jin J, Yang YP, Bian Z, Chen Z, Fan MW. Enhanced immunogenicity of an anti-caries vaccine encoding a cell-surface protein antigen of Streptococcus mutans by intranasal DNA prime-protein boost immunization. J Gene Med 2009; 11: 1039-47.

21 Fraud S, Maillard JY, Kaminski MA, Hanlon GW. Activity of amine oxide against biofilms of Streptococcus mutans: a potential biocide for oral care formulations. J Antimicrob Chemother 2005; 56: 672-7.

22 Hamada S, Koga T, Ooshima T. Virulence factors of Streptococcus mutans and dental caries prevention. J Dent Res 1984; 63: 407-11.

23 Loesche WJ. Role of Streptococcus mutans in human dental decay. Microbiol Rev 1986; 50: 353-80.

24 Jefferson KK. What drives bacteria to produce a biofilm? FEMS Microbiol Lett 2004; 236: 163-73.

25 Al-Hashimi I, Levine MJ. Characterization of in vivo salivary-derived enamel pellicle. Arch Oral Biol 1989; 34: 289-95.

26 Liljemark WF, Bloomquist C. Human oral microbial ecology and dental caries and periodontal diseases. Crit Rev Oral Biol Med 1996; 7: 180-98.

27 Jakubovics NS, Stromberg N, van Dolleweerd CJ, Kelly CG, Jenkinson HF. Differential binding specificities of oral streptococcal antigen I/II family adhesins for human or bacterial ligands. Mol Microbiol 2005; 55: 1591-605.

28 Jenkinson HF, Lamont RJ. Streptococcal adhesion and colonization. Crit Rev Oral Biol Med 1997; 8: 175-200.

29 Marsh PD. Are dental diseases examples of ecological catastrophes? Microbiology 2003; 149: 279-94.

30 Bowen WH, Pearson SK, Young DA. The effect of desalivation on coronal and root surface caries in rats. J Dent Res 1988; 67: 21-3.

31 Douglas CW, Russell RR. The adsorption of human salivary components to strains of the bacterium Streptococcus mutans. Arch Oral Biol 1984; 29: 751-7.

32 Hajishengallis G, Nikolova E, Russell MW. Inhibition of Streptococcus mutans adherence to saliva-coated hydroxyapatite by human secretory immunoglobulin A (S-IgA) antibodies to cell surface protein antigen I/II: reversal by IgA1 protease cleavage. Infect Immun 1992; 60: 5057-64.

33 Danielsson Niemi L, Hernell O, Johansson I. Human milk compounds inhibiting adhesion of mutans streptococci to host ligand-coated hydroxyapatite in vitro. Caries Res 2009; 43: 171-8.

34 Vacca-Smith AM, Van Wuyckhuyse BC, Tabak LA, Bowen WH. The effect of milk and casein proteins on the adherence of Streptococcus mutans to saliva-coated hydroxyapatite. Arch Oral Biol 1994; 39: 1063-9.

35 Bollinger RR, Everett ML, Palestrant D, Love SD, Lin SS, Parker W. Human secretory immunoglobulin A may contribute to biofilm formation in the gut. Immunology 2003; 109: 580-7.

36 Smith DJ, Mattos-Graner RO. Secretory immunity following mutans streptococcal infection or immunization. Curr Top Microbiol Immunol 2008; 319: 131-56.

37 Jespersgaard C, Hajishengallis G, Russell MW, Michalek SM. Identification and characterization of a nonimmunoglobulin factor in human saliva that inhibits Streptococcus mutans glucosyltransferase. Infect Immun 2002; 70: 1136-42. 